\title{
Percutaneous Endoscopic Lumbar Discectomy Versus Microdiscectomy for the Treatment of Lumbar Disc Herniation: Pain, Disability, and Complication Rate-A Randomized Clinical Trial
}

\author{
GUILHERME MEYER, MD, MS, ${ }^{1,2}$ IVAN DIAS DA ROCHA, MD, MS, ${ }^{1}$ \\ ALEXANDRE FOGAÇA CRISTANTE, MD, PhD,${ }^{1}$ RAPHAEL MARTUS MARCON, MD, PhD, ${ }^{1}$ \\ THIAGO PEREIRA COUTINHO, MD, MS, ${ }^{1}$ ALESSANDRO GONZALEZ TORELLI, MD, ${ }^{1}$ \\ PEDRO ARAUJO PETERSEN, MD, MS, ${ }^{1}$ OLAVO BIRAGHI LETAIF, MD, PhD, ${ }^{1}$ \\ TARCÍSIO ELOY PESSOA DE BARROS FILHO, MD, PhD ${ }^{1}$ \\ ${ }^{l}$ Spine Surgery Division, Instituto de Ortopedia e Traumatologia da Faculdade de Medicina da Universidade de São Paulo, FMUSP, São Paulo, Brazil, \\ ${ }^{2}$ Spine Surgery Division, Instituto Vita, São Paulo, Brazil
}

\begin{abstract}
Purpose: The objective was to compare the traditional microdiscectomy with percutaneous endoscopic lumbar discectomy for the treatment of disc herniations regarding pain, disability, and complications.

Methods: Randomized clinical trial with 47 patients with disc herniations treated with 2 different surgical techniques: traditional microdiscectomy or percutaneous endoscopic lumbar discectomy. Forty-seven patients were divided into 2 groups and monitored for 12 months. Irradiated and low back pain were evaluated with the visual analog scale. Surgery complications were recorded.

Results: After surgery, the sciatica and disability improved significantly but without significant differences between the groups. Improvements in back pain were significant until the third month. There were no statistical differences between groups regarding recurrence, infection, and the need for reoperation.

Conclusions: Endoscopic discectomy results are similar to those of conventional microdiscectomy regarding pain and disability improvement. Postoperative lumbar pain is less intense with endoscopic discectomy than conventional microdiscectomy only during the first 3 months. Endoscopic discectomy is a safe and efficient alternative to microdiscectomy.

Clinical Trials: Trial protocol registration number: RBR-5symrd (http://www.ensaiosclinicos.gov.br).
\end{abstract}

Endoscopic Minimally Invasive Surgery

Keywords: intervertebral disc displacement, diskectomy, percutaneous, endoscopy, prospective studies, intervertebral disc, microsurgery

\section{INTRODUCTION}

Microdiscectomy (MD) is considered the gold standard surgical option for the treatment of lumbar disc herniations. However, there is still concern regarding the risks of damage to muscles, such as the multifidus, and exaggerated resection of the articular facet during laminectomy, causing instability, formation of extensive epidural fibrosis, and the continuation of radiated pain in addition to the possibility of surgical site infection. Endoscopic discectomy (ED) has been proposed as a less invasive alternative. ${ }^{1-5}$

A few prospective randomized clinical trials comparing traditional $\mathrm{MD}$ with $\mathrm{ED}^{1-5}$ found no significant differences between groups regarding pain and function after surgery. ${ }^{3,4}$ However, ED resulted in shorter hospitalization times, less bleeding, lower inflammatory serum markers, ${ }^{5}$ and reductions in pain and complication rates. ${ }^{5}$

Due to the low number of published prospective randomized clinical trials, we felt it was necessary to conduct this kind of study. The main objective of this study is to compare traditional MD with ED in symptom relief in a 12-month follow-up. The secondary objectives are to evaluate symptom recurrence, complications, and the need for reoperation. The hypothesis was that one technique (the ED technique) would be superior to the other for pain and disability outcomes after surgery. 


\section{METHODS}

\section{Study Design, Setting, and Ethics}

A randomized clinical trial was conducted to compare 2 surgical approaches to the treatment of disc herniation of the lumbar spine that is refractory to clinical treatment. The patients were recruited in the spine diseases outpatient department of a public university hospital and signed informed consent forms. The study protocol was approved by the local ethics committee, and the study protocol was registered in a clinical trials registration database (number omitted for blinding purposes). All included patients signed the informed consent form.

This trial did not receive any type of funding. Patients were operated within the public health care system. The authors are all public servants (university teachers and/or assistant surgeons working in the public hospital), and they have no conflicts of interest to disclose.

\section{Participants}

Adult patients (18-70 years old) with surgical indication were included (magnetic resonance imaging [MRI] in concordance with clinical symptoms and failure of at least 6 weeks of conservative treatment).

The study was restricted to cases requiring a simple endoscopic approach without the need of a foraminotomy or bone work. Cases in which the endoscopic approach was not possible or that would require bone resection were excluded. Other exclusion criteria were cauda equina syndrome, strength deficit characterized by strength equal to or lower than III/V, bone stenosis of the canal or of the lateral recess associated with disc herniation, foraminal stenosis, previous surgery on the lumbar vertebral spine, the presence of 2 or more symptomatic disc herniations, and any other disease or medication that would be a contraindication for surgery.

\section{Interventions}

This study compared percutaneous ED and MD using the techniques described in detail below. All procedures were performed at the same surgical center between October 2013 and September 2015 with the patient under general anesthesia. The ED were performed by only 2 doctors with experience in more than 30 cases. The MD were performed by the clinical staff of the spine group assisted by medical residents.

\section{Percutaneous ED: Surgical Technique}

A multichannel endoscope consisting of a set of forceps and specific instruments used for spine surgery were used in all the endoscopic procedures of this study. There are 2 possible endoscopic approaches for direct radicular decompression: the classic $^{6}$ transforaminal (TF) approach and the interlaminar (IL) approach. Elevated iliac crests, the angulation of sacral inclination, and foraminal anatomy in L5-S1 are all anatomical barriers to TF access in this level when a bur is not used. Furthermore, there is a very wide window between the laminae of L5 and S1 and a dural sac with less concentration of roots, enabling an IL approach. The choice for IL or TF in this study depended on the surgeon's preference for each case.

The approach is performed with the patient in decubitus ventral, with the hips semiflexed to decrease lumbar lordosis. This promotes slight lumbar flexion and increases the foraminal and IL space.

Guided by radioscopy, the disc space is punctured with a needle to provide spinal access through the Kambin's safe triangle. A discography is performed, with $2 \mathrm{~mL}$ of methylene blue solution, nonionic contrast, and physiological serum at a ratio of $1: 2: 2$. This discography enables visualization of the disc degeneration, while the methylene blue aids differentiation of the structures, as it dyes the disc and the herniation but not the nerve root or the dural sac.

A guide wire and then a dilator of $6.9 \mathrm{~mm}$ in diameter is introduced into the disc space. Next, a cannula with an external diameter of $8 \mathrm{~mm}$ is introduced over the dilator, and then comes the endoscope inside this cannula. The positioning is extremely important because adequate decompression depends on correct positioning. An image intensifier is used so that the instruments can be placed near the disc herniation and in a safe manner.

The endoscope allows visualization of the disc herniation and spinal canal structures. Forceps and other instruments are introduced through the working canal for removal of the disc herniation and radicular decompression (Figure 1). Direct visualization of the free nerve root and the presence 


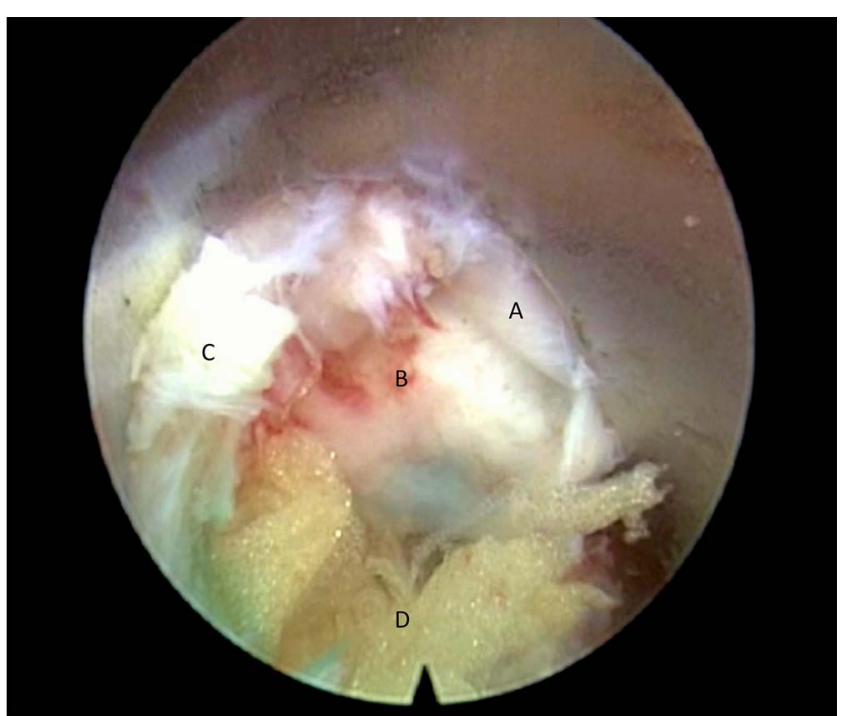

Figure 1. Endoscopic view of a right-side L5-S1 foraminal herniation. A: exiting nerve root; $\mathrm{B}$ : extruded disc fragment; $\mathrm{C}$ : flavum ligament on the dorsal limit of the foramen; $D$ : foraminal fat.

of pulsation of the epidural structures are signs of adequate decompression.

For the IL approach, an image intensifier is used to obtain AP images that show the IL window. A dilator is introduced through a 7-mm incision next to the spinous process and supraspinous ligament, ipsilateral to the herniation. It is inserted until it is supported dorsally by the ligamentum flavum and laterally by the medial edge of the facet joint. The cannula is introduced over this dilator and then the endoscope. Under direct vision, the ligamentum flavum is opened, enabling the entry of the endoscope inside the vertebral canal. With the entry of endoscope inside the canal, it is possible to move apart the nerve structures, enabling the removal of the herniation and radicular decompression (Figure 2).

The patients are discharged depending on the level of pain and independence to walk and use the bathroom without assistance, which occurred 1 day after the procedure in all cases.

\section{$M D$}

MD was performed in the conventional way, using magnifying loupes or a surgical microscope. A conservative laminotomy and removal of the ligamentum flavum are performed in order to enter the vertebral canal, remove disc herniation, and decompress the nerve root.

The patients are discharged 1 or 2 days after the procedure using the same criteria.

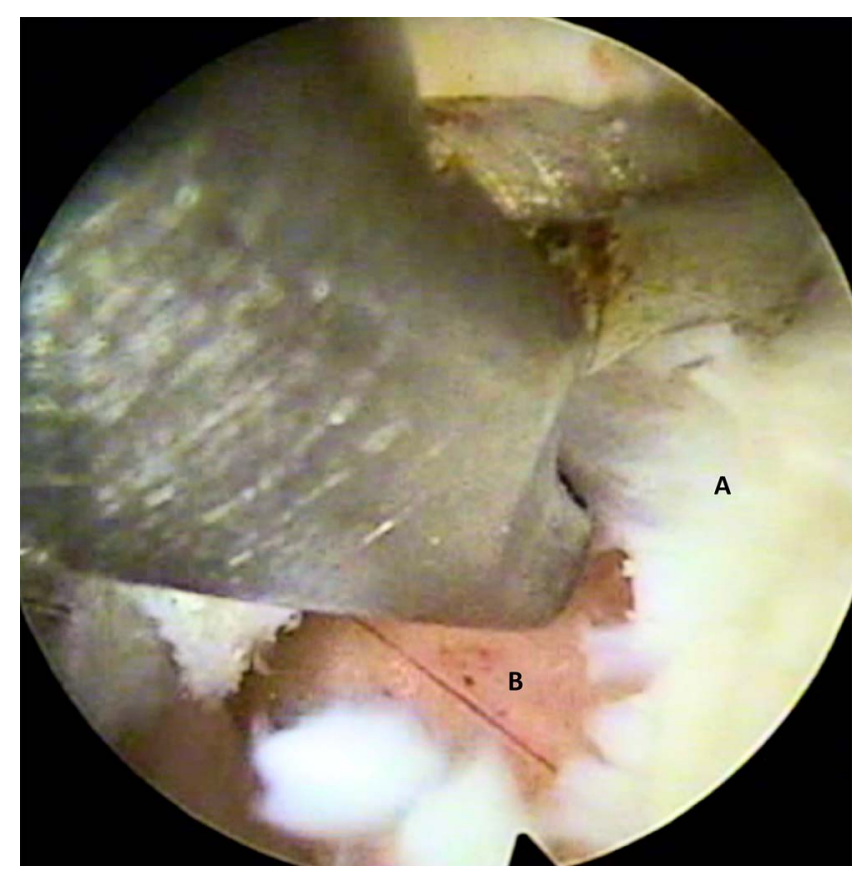

Figure 2. Endoscopic view of the opening of the flavum ligament. A: flavum ligament; B: epidural fat beneath the flavum ligament.

\section{Outcomes}

Initially, in this study, the patients were evaluated preoperatively using MRI images to determine the position of the herniation and the presence and degree of migration and/or sequestration, according to the classification of Lee et $\mathrm{al}^{7}$ for herniation zones. The main outcomes evaluated were pain and disability, as described below.

The patients were followed up for 12 months; during this period, records were kept of recurrence of disc herniation, postoperative complications (fistulas and infections), and the need for a new surgical intervention. Intraoperative complications were also documented, such as accidental lesion of the dura mater.

Pain was evaluated by the visual analog scale (VAS) and was applied separately to the lumbar region and lower limb to which the pain had radiated. We considered it important to measure lumbar pain once it can be important postoperatively according to surgical tissue damage.

The assessments of pain and disability were performed by an evaluator who was independent of the surgical team and who was not told which surgical approach had been used. Pain and disability were assessed using the VAS and the Oswestry Disability Index (ODI), respectively, preoperatively and at 1 week and 1, 3, 6, and 12 months after 
Table 1. Baseline data for the 2 study groups. ${ }^{\text {a }}$

\begin{tabular}{lcc}
\hline Variable & MD Group & ED Group \\
\hline Number of patients & 24 & 23 \\
Age (y) & $45.2(10.6)$ & $47.2(10.6)$ \\
Sciatica: VAS score & $8.7(1.4)$ & $8.4(1.7)$ \\
Lumbar pain: VAS score & $6.5(2.6)$ & $5.4(2.6)$ \\
ODI & $29.0(8.8)$ & $28.9(10.0)$
\end{tabular}

Abbreviations: MD, microdiscectomy; ED, endoscopy; VAS, visual analog scale; ODI, Oswestry Disability Index.

${ }^{\mathrm{a}}$ Values are shown as mean (SD).

surgery. The assessments were conducted face-toface or by telephone.

\section{Sample Size, Randomization, Allocation Ratio, and Blinding}

For the calculation of sample size, we used the following premises. Primary outcome was reduction in pain (VAS), assuming that a variation of more than 2 points indicates a difference between groups. We considered 2 points as the SD, and we assumed a possible loss to follow-up of $10 \%$ of the sample. Thus, for the study to have $80 \%$ power to detect a minimum difference of 2 points on the VAS and assuming a type I error of $5 \%(P<.05), 20$ patients would be needed in each group.

The patient randomization was done by drawing a closed, sealed envelope during the scheduling of the surgical procedure by the department secretary, with patients being assigned to each group at a ratio of $1: 1$. Due to the differences between procedures, such as incision size, it was not possible to blind the patients to which procedure was being carried out.

\section{Statistical Analysis}

For the comparison between groups and between preoperative and postoperative phases, the Wilcoxon and Mann-Whitney $U$ tests were used. For the long-term assessment, analysis of variance was used. A probability of less than $5 \%(P<.05)$ was assumed to be statistically significant.

\section{RESULTS}

\section{Baseline Characteristics}

Forty-seven patients were enrolled. They were randomly divided into 2 groups: $23(48.9 \%)$ in the ED group and $24(51.91 \%)$ in the MD group. There was no statistical difference between groups regarding baseline data for age or pain scores (Table 1; $P>.05$ for all). The study involved 29 men and 18
Table 2. Vertebral levels treated per group.

\begin{tabular}{lcc}
\hline Level & MD Group & ED Group \\
\hline L1-L2 & 0 & 1 \\
L2-L3 & 0 & 0 \\
L3-L4 & 2 & 2 \\
L4-L5 & 10 & 8 \\
L5-S1 & 12 & 12 \\
Total & 24 & 23 \\
\hline
\end{tabular}

Abbreviations: MD, microdiscectomy; ED, endoscopy.

women (14:9 men in the ED group and 16:8 women in the MD group).

The operated levels among the patients submitted to ED and MD are described in Table 2. In the ED group, there were $15 \mathrm{TF}$ and $8 \mathrm{IL}$ approaches. The level L5-S1 received all 8 IL approaches and other 4 TF.

\section{Postoperative Outcomes}

Pain was measured again 1 week after surgery and then at 1, 3, 6, and 12 months. The results, shown in Table 3, demonstrate a gradual and significant reduction in pain for both groups when compared with the preoperative assessment $(P<.05)$. The figures represent an improvement of $61 \%$ over 7 days. The improvement in radiated pain to the leg was maintained throughout the 12month follow-up. At 12 months, we found an average improvement of $68 \%$, without statistical difference between the groups. Over time, the clinical improvement seen immediately after surgery was maintained, with no one approach being significantly better than the other. At the seventh day of follow-up, we found a reduction in average

Table 3. Lumbar pain and sciatica before and after the treatment. ${ }^{a}$

\begin{tabular}{lcccc}
\hline & MD Mean & ED Mean & $\boldsymbol{P}$ & Total Mean \\
\hline Sciatica & & & & \\
$\quad$ Preoperative & $8.7(1.4)$ & $8.4(1.7)$ & .421 & $8.5(1.9)$ \\
Week 1 & $3.3(2.3)$ & $3.5(3.4)$ & .905 & $3.3(2.8)$ \\
Month 1 & $2.8(2.9)$ & $3.3(2.9)$ & .318 & $2.9(2.8)$ \\
Month 3 & $2.8(3.1)$ & $1.7(1.8)$ & .161 & $2.4(2.7)$ \\
Month 6 & $2.3(3.0)$ & $2.5(2.5)$ & .648 & $2.4(2.7)$ \\
Month 12 & $3.0(3.6)$ & $2.1(1.9)$ & .958 & $2.6(2.8)$ \\
$P$ & $<.001^{\mathrm{c}}$ & $<.001^{\mathrm{c}}$ & $<.001^{\mathrm{c}}$ & \\
Lumbar pain & & & & \\
Preoperative & $6.5(2.6)$ & $5.4(2.6)$ & .081 & $6.0(2.6)$ \\
Week 1 & $4.4(2.1)$ & $2.3(2.8)$ & $.001^{\mathrm{b}}$ & $3.4(2.7)$ \\
Month 1 & $3.7(2.2)$ & $1.7(2.2)$ & $.003^{\mathrm{b}}$ & $2.7(2.4)$ \\
Month 3 & $3.3(2.4)$ & $1.6(2.0)$ & $.009^{\mathrm{b}}$ & $2.5(2.4)$ \\
Month 6 & $2.8(2.6)$ & $2.4(3.0)$ & .385 & $2.6(2.8)$ \\
Month 12 & $2.4(2.5)$ & $2.5(2.5)$ & .835 & $2.4(2.5)$ \\
$P$ & $<.001^{\mathrm{c}}$ & $<.001^{\mathrm{c}}$ & $<.001^{\mathrm{c}}$ & \\
\hline
\end{tabular}

Abbreviations: MD, microdiscectomy; ED, endoscopy.

${ }^{\text {a }}$ Values are shown as mean (SD).

${ }^{\mathrm{b}}$ Statistically significant between groups.

${ }^{\mathrm{c}}$ Statistically significant between follow-up periods. 


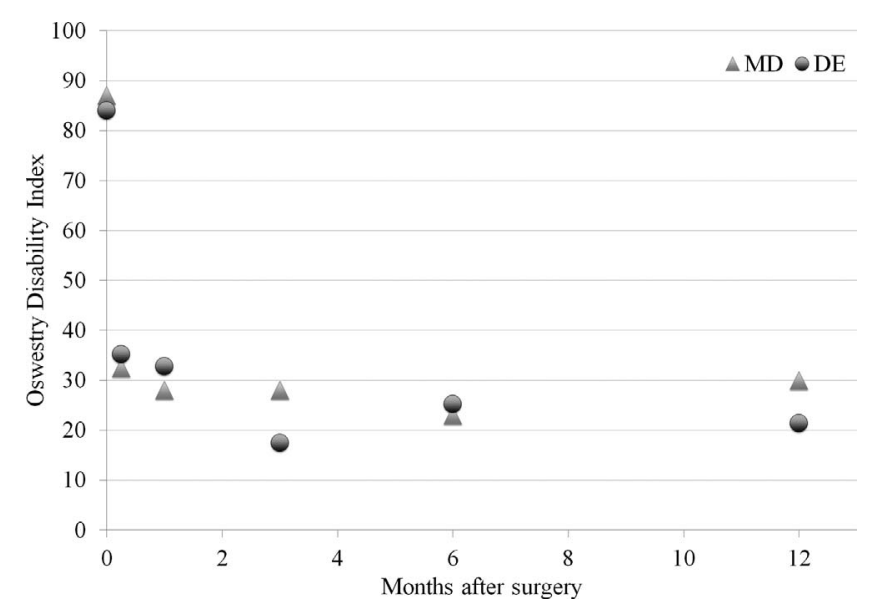

Figure 3. Function as measured by the Oswestry Disability Index (ODI) before and after surgery for herniated disc treatment in patients submitted to microdiscectomy (MD) or endoscopic discectomy (ED). Values are shown as the mean scores in each follow-up point. $P>.05$ for comparisons between the study groups.

lumbar VAS values (ie, at the site of surgery) for both ED and MD, with a statistical difference between the groups $(P<.001)$, as shown in Table 3 . Lumbar pain was lower in ED than in MD until the third postoperative month. From 6 months after surgery, the difference between groups was no longer significant.

Before surgery, patients had intense functional disability, according to the ODI. This was significantly reduced 1 week after surgery $(P<.05)$, but without statistical differences between the groups after that (Figure 3). Analysis of variance for repeated measurements demonstrated a significant improvement in disability between the preoperative assessments and the assessment at 7 days after surgery, but there was no difference between the other assessments. However, the groups presented a significant improvement in disability that remained unchanged throughout the 12 months of follow-up.

\section{Complications}

During 1 MD procedure, 1 dural lesion was identified that was repaired during the same surgical procedure, without any further consequences. No dural lesions were identified or treated in the ED group.

There was 1 postoperative infection in the MD group. No infections were observed in the ED group.

There were 3 recurrences: 1 in the ED group and 2 in the MD group. Symptoms persisted following surgery in 2 patients in the ED group, and a new MRI was performed that revealed the presence of a disc fragment showing an incomplete decompression maintaining root compression. These 2 patients underwent an MD, with improvement of symptoms. Both patients with incomplete endoscopic decompression were submitted to a TF procedure at level L5-S1. Thus, 6 complementary surgical procedures were performed during the follow-up of these patients: 3 in the ED group ( 2 incomplete and 1 recurrence) and 3 in the MD group (2 recurrences and 1 infection).

\section{DISCUSSION}

This randomized clinical study showed that the ED technique is safe and effective and offers similar results to those found in the literature ${ }^{1,3,7-10}$ and also is as good as MD in terms of improvements in disability and pain. However, less low back pain was confirmed in the ED group at 7 days, 1 month, and 3 months following surgery when compared to the MD group.

Percutaneous and endoscopic minimally invasive surgeries have been rapidly evolving, ${ }^{11,12}$ with the aim to provide lower complications rates while delivering comparable clinical outcomes and lower readmission rates, ${ }^{13}$ reduced overall costs, ${ }^{13-15}$ safety and efficacy even in risky populations, ${ }^{16,17}$ and with the possibility to perform surgery under local anesthesia. ${ }^{18,19}$ Reduced postoperative lumbar pain is attributed to the minimal lesion in healthy tissues, less bleeding, and the absence of muscle retraction in the ED. Besides requiring a larger incision, MD also involves retraction of the paravertebral muscles, bone resection of part of the lamina and the medial edge of the facet joint, and partial removal of the ligamentum flavum.

The reduction in postoperative pain plays an important role in reduced hospital stays and early patient rehabilitation. The manipulation of nerve structures is also reduced in ED. ${ }^{1}$ There may be a 3.2 times higher chance of continuation of the symptoms in the presence of extensive epidural fibrosis, ${ }^{20}$ which is related to the continuation of sciatic pain and unsatisfactory surgical outcomes and should therefore be prevented. ${ }^{20-22}$

In our study, we had 2 patients with incomplete decompression by the endoscopic approach, both of whom underwent a TF approach in L5-S1. As mentioned, this segment presents anatomical barriers that often make a suitable $\mathrm{TF}$ approach but difficult without a bur. ${ }^{23} \mathrm{We}$ attribute this fact to the choice of the TF approach in these 2 patients. 
An IL approach would probably have resulted in satisfactory outcomes that would have led to the ED group having only a single revision surgery, while the MD group had 3. We emphasize the importance of surgical planning and of the correct indication of each approach for the success of the technique.

This study identified 3 recurrences during the 12month follow-up: 1 in the ED group and 2 in the MD group. The patients who presented recurrent herniations had a period of time free from symptoms and then began to have sciatica again, and the recurrence of disc herniation was confirmed by a new MRI. Because of the small size of the groups, we could not make any assertions about the lower incidence of recurrence in the endoscopic approach.

Accidental lesions of the dura mater, though infrequent in MD, can lead to neurological deterioration, cerebrospinal fluid leakage, and even meningitis if not properly managed. ${ }^{24,25}$ In the $\mathrm{ED}$, dura mater lesions are very rare, and due to the absence of cavities created by the surgical procedure itself, they can have a favorable evolution. Due to the constant positive pressure of the saline administered during the procedure, it was not possible to detect leakage of spinal fluid or the emergence of a nerve root due to a possible defect created in the dura mater, making an analysis of accidental lesions incidence difficult.

Postoperative infections in spinal surgery are severe adverse events potentially extending to the central nervous system; osteomyelitis and long-term antibiotic treatment are all associated with this complication. ${ }^{26-28}$ ED offers several advantages: it is a less aggressive surgical procedure, it does not form "dead space," and it is performed with constant irrigation (lavage). As a result, the rates of infection are much lower. ${ }^{29-33}$ In addition, it is possible to add antibiotics to the physiological saline used.

\section{Limitations}

One of the limitations is the reduced sample size. In a larger study, differences between groups might be clearer. Another limitation is that ED surgeries were performed by 2 experienced surgeons, while MD were performed by experienced surgeons, with the assistance of residents, and this may have influenced the results. A study that does not involve the participation of residents and a smaller number of surgeons would provide greater scientific rigor.

\section{CONCLUSIONS}

The clinical results of ED are similar to those of MD in regard to improvement in radiated pain and disability but offer an advantage in relation to postoperative lumbar pain up to the third month. ED is a safe and effective technique, representing an alternative to the gold standard of MD.

\section{REFERENCES}

1. Ruetten S, Komp M, Merk H, Godolias G. Fullendoscopic interlaminar and transforaminal lumbar discectomy versus conventional microsurgical technique: a prospective, randomized, controlled study. Spine (Phila Pa 1976). 2008;33(9):931-939. doi:10.1097/BRS.0b013e31816c8af7.

2. Mayer HM, Brock M. Percutaneous endoscopic discectomy: surgical technique and preliminary results compared to microsurgical discectomy. J Neurosurg. 1993;78(2):216-225. doi:10.3171/jns.1993.78.2.0216.

3. Hermantin FU, Peters T, Quartararo L, Kambin P. A prospective, randomized study comparing the results of open discectomy with those of video-assisted arthroscopic microdiscectomy. J Bone Joint Surg Am. 1999;81(7):958-965. doi:10. 1016/S0161-4754(00)90127-1.

4. Pan L, Zhang P, Yin Q. Comparison of tissue damages caused by endoscopic lumbar discectomy and traditional lumbar discectomy: a randomised controlled trial. Int J Surg. 2014;12(5):534-537. doi:10.1016/j.ijsu.2014.02.015.

5. Pan Z, Ha Y, Yi S, Cao K. Efficacy of transforaminal endoscopic spine system (TESSYS) technique in treating lumbar disc herniation. Med Sci Monit. 2016;22:530-539. doi:10.12659/MSM.894870.

6. Kambin P, Brager MD. Percutaneous posterolateral discectomy. Anatomy and mechanism. Clin Orthop Relat Res. Oct. 1987;(223):145-154. http://www.ncbi.nlm.nih.gov/pubmed/ 3652568. Accessed February 21, 2019.

7. Lee S, Kim SK, Lee SH, et al. Percutaneous endoscopic lumbar discectomy for migrated disc herniation: classification of disc migration and surgical approaches. Eur Spine $J$. 2007;16(3):431-437. doi:10.1007/s00586-006-0219-4.

8. Andersson GB, Brown MD, Dvorak J, et al. Consensus summary of the diagnosis and treatment of lumbar disc herniation. Spine (Phila Pa 1976). 1996;21(suppl 24):75S-78S.

9. Choi G, Lee S-H, Bhanot A, Raiturker PP, Chae YS. Percutaneous endoscopic discectomy for extraforaminal lumbar disc herniations. Spine (Phila Pa 1976). 2007;32(2):E93-E99. doi:10.1097/01.brs.0000252093.31632.54.

10. Ruetten S, Komp M, Godolias G. An extreme lateral access for the surgery of lumbar disc herniations inside the spinal canal using the full-endoscopic uniportal transforaminal approach-technique and prospective results of 463 patients. Spine (Phila Pa 1976). 2005;30(22):2570-2578.

11. Yoon JW, Wang MY. The evolution of minimally invasive spine surgery. Spine J. 2019;30:149-158.

12. Yeung AT. Foreword, percutaneous and endoscopic MIS special issue. Int J Spine Surg. 2014;8:14. doi:10.14444/1014.

13. Lewandrowski K-U. Incidence, management, and cost of complications after transforaminal endoscopic decompression surgery for lumbar foraminal and lateral recess stenosis: a value 
proposition for outpatient ambulatory surgery. Int $J$ Spine Surg. 2019;13(1):53-67. doi:10.14444/6008.

14. Wang MY, Lerner J, Lesko J, McGirt MJ. Acute hospital costs after minimally invasive versus open lumbar interbody fusion. J Spinal Disord Tech. 2012;25(6):324-328. doi:10.1097/BSD.0b013e318220be32.

15. Choi K-C, Shim H-K, Kim J-S, et al. Cost-effectiveness of microdiscectomy versus endoscopic discectomy for lumbar disc herniation. Spine J. Feb 2019. doi:10.1016/j.spinee.2019.02. 003.

16. Bae JS, Lee S-H. Transforaminal full-endoscopic lumbar discectomy in obese patients. Int J Spine Surg. 2016;10:18. doi:10.14444/3018.

17. Rieger B, Sitoci-Ficici KH, Reinshagen C, et al. Endoscopic and microscopic segmental decompression via translaminar crossover spinal approach in elderly patients. World Neurosurg. Jan 2019. doi:10.1016/j.wneu.2019.01.078.

18. Gore S, Yeung A. The "inside out" transforaminal technique to treat lumbar spinal pain in an awake and aware patient under local anesthesia: results and a review of the literature. Int J Spine Surg. 2014;8:28. doi:10.14444/1028.

19. Yeung A, Gore S. Endoscopic foraminal decompression for failed back surgery syndrome under local anesthesia. Int $J$ Spine Surg. 2014;8:22. doi:10.14444/1022.

20. Ross JS, Robertson JT, Frederickson RCA, et al. Association between peridural scar and recurrent radicular pain after lumbar discectomy: magnetic resonance evaluation. Neurosurgery. 1996;38(4):855-861.

21. Coskun E, Suzer T, Topuz O, Zencir M, Pakdemirli E, Tahta K. Relationships between epidural fibrosis, pain, disability, and psychological factors after lumbar disc surgery. Eur Spine J. 2000;9(3):218-223. doi:10.1007/s005860000144.

22. Geisler F. Prevention of peridural fibrosis: current methodologies. Neurol Res. 1999;21(suppl 1):S9-S22. doi:10. 1080/01616412.1999.11741021.

23. Choi KC, Park C-K. Percutaneous endoscopic lumbar discectomy for L5-S1 disc herniation: consideration of the relation between the iliac crest and L5-S1 disc. Pain Physician. 2016;19(2):E301-E308.

24. Espiritu MT, Rhyne A, Darden B V. Dural tears in spine surgery. J Am Acad Orthop Surg. 2010;18(9):537-545.

25. Guerin P, El Fegoun AB, Obeid I, et al. Incidental durotomy during spine surgery: incidence, management and complications. A retrospective review. Injury. 2012;43(4):397401. doi:10.1016/j.injury.2010.12.014.

26. Raiszadeh R, Rhines LD. Management of thoracic spine infections. Oper Tech Neurosurg. 2004;7(4):199-205. doi:10. 1053/j.otns.2005.06.007.

27. Rihn JA, Lee JY, Ward WT. Infection after the surgical treatment of adolescent idiopathic scoliosis. Spine (Phila Pa 1976). 2008;33(3):289-294. doi:10.1097/BRS.0b013e318162016e.

28. Meyer GPC, Gomes FCP, Lima ALLM, et al. Estudo retrospectivo das infecções pós-operatórias em cirurgia de coluna: correlação com o número de limpezas cirúrgicas realizadas. Coluna/Columna. 2011;10(2):127-131. doi:10.1590/ S1808-18512011000200009.

29. Olsen MA, Nepple JJ, Riew KD, et al. Risk factors for surgical site infection following orthopaedic spinal operations. $J$ Bone Jt Surgery (Am. Vol). 2008;90(1):62-69. doi:10.2106/JBJS. F.01515.

30. Fei Q, Li J, Lin J, et al. Risk factors for surgical site infection after spinal surgery: a meta-analysis. World Neurosurg. 2016;95:507-515. doi:10.1016/j.wneu.2015.05.059.

31. Ee WWG, Lau WLJ, Yeo W, Von Bing Y, Yue WM. Does minimally invasive surgery have a lower risk of surgical site infections compared with open spinal surgery? Clin Orthop Relat Res. 2013;472(6):1718-1724. doi:10.1007/s11999-0133158-5.

32. Li XC, Zhong CF, Deng GB, Liang RW, Huang CM. Full-endoscopic procedures versus traditional discectomy surgery for discectomy: a systematic review and meta-analysis of current global clinical trials. Pain Physician. 2016;19(3):103118.

33. Choi G, Pophale CS, Patel B, Uniyal P. Endoscopic spine surgery. J Korean Neurosurg Soc. 2017;60(5):485-497. doi:10.3340/jkns.2017.0203.004.

Disclosures and COI: The authors have no competing interests to declare. There is no funding source. This article was revised and approved by an institutional review board. Informed consent was obtained from all individual participants included in the study.

Corresponding Author: Guilherme Meyer, R. Dr. Ovídio Pires de Campos, 333 Cerqueira César, São Paulo, SP 05403-010, Brazil. Phone: +55 11 94187-8350; Email: gpcmeyer@gmail.com.

Published 29 February 2020

This manuscript is generously published free of charge by ISASS, the International Society for the Advancement of Spine Surgery. Copyright (C) 2020 ISASS. To see more or order reprints or permissions, see http://ijssurgery.com. 